\title{
How Supervisor-Subordinate Guanxi Influence Employee Innovative Behavior: A Moderated Mediation Model
}

Yu Gao

\section{Haiyan Liu (D)}

School of Economics and Management, China University of Geosciences (Beijing), Beijing, People's Republic of China
Correspondence: Haiyan Liu

Email liuhy@cugb.edu.cn
Purpose: In the practice of inspiring employees' innovative behavior (IB), managers often pay attention to the role of policies, capitals, incentive measures, equipment and other factors, while ignoring the role of the relationship between leaders and employees. Based on social exchange theory and conservation of resources theory, this paper is to examine the mediating role of job satisfaction (JS) and organizational commitment (OC) in the relationship between supervisorsubordinate guanxi (SSG) and employee IB, and the moderating role of psychological safety (PS) in the series mediation model between SSG and employee IB.

Patients and Methods: Cross-sectional data came from 207 employees of Chinese scientific and technological enterprises. The participants completed the SSG scale, JS scale, OC scale, IB scale, and PS scale. SPSS PROCESS macro was used to test the research hypothesis.

Results: SSG was positively associated with employee IB. SSG can not only influence IB through JS or OC (SSG $\rightarrow \mathrm{JS} \rightarrow \mathrm{IB} ; \mathrm{SSG} \rightarrow \mathrm{OC} \rightarrow \mathrm{IB})$ but also influence IB through JS and OC $(\mathrm{SSG} \rightarrow \mathrm{JS} \rightarrow \mathrm{OC} \rightarrow \mathrm{IB})$. PS moderated the joint mediating effects between SSG and IB $(\mathrm{SSG} \rightarrow \mathrm{JS} \rightarrow \mathrm{OC} \rightarrow \mathrm{IB})$.

Conclusion: To stimulate employees' IB within an enterprise can pay attention to the relationship between leaders and employees, improve employee JS, and strengthen employee OC. The lower the PS, the stronger the joint mediation effects between SSG and employee IB. Therefore, leaders should also pay attention to employees with high PS to avoid the counterproductive effect of cultivating SSG.

Keywords: supervisor-subordinate guanxi, job satisfaction, organizational commitment, innovative behavior, psychological safety

\section{Introduction}

The outbreak of COVID-19 in early 2020 has led to increased downward pressure on the global economy. The recent rapid spread of mutant strains such as Delta and Lambda makes the global economic recovery still fragile. Facing this complex and uncertain external challenge, many small and medium-sized enterprises were forced to stop production. Such severe situation forces enterprises to rethink their management mode in all aspects so as to expand living space, get out of enterprise dilemmas, and win market opportunities. In the complex and changeable environment, it is inevitable for an enterprise to maintain its survival and development, enhance its competitive advantage to break the stereotype of thinking in a timely manner and develop innovative ideas. ${ }^{1-3}$ As the main body of innovative activities, 
employees can continue to stimulate their innovative vitality to better help enterprises improve quality and efficiency. Therefore, stimulating the innovative behavior (IB) of employees has become the focus of theoretical and practical circles.

Employees' IB is a multistage process, which includes the generation, dissemination within the enterprise and final realization of innovative ideas. ${ }^{4}$ In previous management practices, many enterprise managers screened employees with creative personality, ${ }^{5}$ created an organizational innovative climate, ${ }^{6}$ and provided policies, capitals, incentive measures and equipment to stimulate employees' innovative vitality, but the results were minimal. Even if employees' innovative passion was stimulated, it is a difficult problem for enterprise managers to effectively maintain employees' innovative momentum and implement IB. The reason may be ignoring the role of factors such as the support from leaders, ${ }^{7}$ the quality of the relationship between leaders and employees, ${ }^{8}$ and discuss openly, put forward new ideas while make mistakes without being punished. ${ }^{9}$ Both supervisor-subordinate guanxi (SSG) and leader-member exchange (LMX) emphasizes the quality of the relationship between leaders and employees. The difference between the two is that the SSG originates from the Oriental cultural background, which is a private exchange relationship between leaders and employees that occurs outside the workplace (either related to work or irrelevant to work, such as dinner, home visits and gift-giving after work), while the LMX originates from the Western cultural background, and is strictly limited to the work-related exchange relationship between leaders and employees in the workplace. ${ }^{10,11}$ Due to the cultural context of "authority-oriented" and "relationoriented" in China, ${ }^{11}$ SSG is more representative of Chinese organizations than LMX. Therefore, it is necessary to explore the influence of SSG on employee IB.

Throughout the previous literatures, it can be summarized as the following four aspects: 1) Few studies have examined the influence of SSG on employee IB. Instead, most of them have investigated the influence of SSG on employees' attitudes (job satisfaction (JS), organizational commitment (OC), turnover intention, etc), employees' perceptions (such as trust in supervisor, subordinates' perceptions of justice), employees' behaviors (such as decision-making behavior, organizational citizenship behavior) and employees' mental health (emotional exhaustion and others). For example, Cheung et $\mathrm{al}^{12}$ found that SSG can cultivate employee JS, which in turn makes employees more willing to stay in the organization and enhance their commitment to the organization. SSG was also an important predictor of employees' trust in leaders. ${ }^{13,14}$ Zhang et $\mathrm{al}^{15}$ found that under the condition of fit, close SSG can also increase employees' perception of distributive justice, procedural justice, and interactional justice. Law et $\mathrm{al}^{10}$ found that SSG can affect the Chinese leaders' administrative decisions. SSG was demonstrated to play an important role in developing employees' prosocial behavior (organizational citizenship behavior). ${ }^{16}$ Charoensukmongkol ${ }^{17}$ found that the relationship between Thai employees and Chinese leaders have had a negative impact on employee emotional exhaustion. 2) Most studies examined the impact of LMX on employees' IB, ${ }^{8,18}$ or directly replace SSG with LMX in China, which fails to effectively highlight the relationship between leaders and employees in the context of Chinese organizations. 3) In the research on the influence of LMX on employees' IB, scholars have found that psychological empowerment, ${ }^{8}$ psychological ownership, ${ }^{19}$ and employee engagement/job commitment ${ }^{20}$ plays an important process mechanism in the relationship between the two. However, most studies were carried out from a single mediator, ignoring the role of 2 or more mediators, in series. 4) In the research on the relationship between SSG and outcome variables, existing studies have found that leadership style, service-oriented organizational citizenship behavior, supervisor job autonomy, and workload levels within the organization moderated the relationship between the two. For example, authoritarian leadership was found to moderate the relationship between SSG and manager voice, and the weaker the authoritarian leadership, the stronger the relationship between the two. ${ }^{21}$ $\mathrm{Wu}$ et $\mathrm{al}^{22}$ found that co-worker service-oriented organizational citizenship behavior moderated leader-member guanxi effect on employees' loafing tendency, and the higher the co-worker service-oriented organizational citizenship behavior, the weaker the relationship between the two. Charoensukmongkol ${ }^{17}$ found that the relationship between SSG and employee emotional exhaustion was positively moderated by Chinese supervisor job autonomy, and negatively moderated by Thai employees' workload levels in their organization. However, there are still few studies on moderating variables that explore the relationship between SSG and outcome variables. Miao et al ${ }^{11}$ also calls for more research on the moderating factors between SSG and outcome variables in the future. Based on the above limitations, this paper will explore whether SSG affects IB of employees and whether this effect is restricted by other factors. 
Social exchange theory holds that the interaction between leaders and employees can be regarded as a social exchange relationship. ${ }^{23}$ Both parties try to exchange with each other based on their respective expectations and goals while believe that their efforts will be compensated or rewarded in some form in the future. ${ }^{23}$ Therefore, this paper believes that employees who receive preferential treatment from leaders should improve their attitudes towards work and make them adopt attitudes and behaviors that are beneficial to their work to repay the preferential treatment given by leaders. Since JS and OC can be regarded as attitudes held under the close SSG, ${ }^{12}$ the improvement or enhancement of them should make employees full of passion at work, ${ }^{24}$ and then urge employees to adopt innovative ways to obtain more performance to repay the organization. Therefore, this paper will separately explore the single mediating role of JS and OC in the relationship between SSG and IB. Since JS also promotes employee OC. ${ }^{12}$ This paper will also explore the indirect effect of SSG on IB through JS and OC in serial. In addition, conservation of resources theory holds that people's resources do not exist independently, but interact and influence each other. ${ }^{25}$ Since psychological safety (PS) is an interpersonal belief formed by mutual respect and trust among team members, it can encourage employees to take interpersonal risks, ${ }^{26}$ which has been proved to be beneficial to employees' $\mathrm{JS}^{27}$ and inspire employees' IB. $^{28}$ Previous research also reported that PS is an important factor in evaluating the influence of leaders on employee behavior. ${ }^{9}$ Based on conservation of resources theory, this paper regards PS as a resource of interpersonal and social relations, ${ }^{29}$ and believes that it may affect the quality of resources that employees obtain from their leaders. When PS is high, employees with close SSG resources are less likely to suffer resource loss, and they are more able to obtain new resources. ${ }^{25}$ When PS is low, employees get relatively few initial resources and are more vulnerable to resource loss, ${ }^{25}$ which is not conducive to the positive role of SSG. Therefore, this paper will further investigate the moderating role of PS in the series mediation model between SSG and employee IB.

To sum up, based on social exchange theory and conservation of resources theory, this paper brings JS and OC into the theoretical model of SSG on employee IB, a series mediation model of SSG on employees' IB through JS and OC was constructed, and the moderating role of PS in the series mediation model was discussed. This paper makes the following contributions to existing research on IB. 1)
At present, few studies have examined the relationship between SSG and employee IB. Instead, most researches were carried out by replacing SSG with LMX. This article supplements and expands the influencing factor that improve employee IB, that is, examines the influence of SSG on employee IB. This variable is different from LMX and is more suitable for Chinese cultural contexts, which can provide a theoretical reference for enterprise management practices. 2) Previous studies on single mediating role often failed to fully understand the complex process mechanism of SSG influencing outcome variables. This paper verifies the complex process mechanism of JS and OC between SSG and employee IB, breaks many mediating thinking of studying a single variable, and explains the formation mechanism of employee IB more comprehensively. 3) No research has examined whether there are moderators in the joint mediation model between SSG and employee IB. In response to the suggestion of Miao et al, ${ }^{11}$ the boundary condition of the influence of SSG on outcome variables is supplemented, and more information for the understanding of SSG on outcome variable is provided. 4) Most previous studies have proved that PS plays a positive role. This paper further confirms whether PS plays a positive or negative role. Whether the higher the PS, the better. Deepening the understanding of the role of PS through this.

\section{Theoretical Background and Hypotheses}

Social exchange theory proposed by Homans, ${ }^{30}$ and explained systematically and comprehensively by Blau, ${ }^{23}$ believing that social exchange is a communication process in which individuals expect to obtain resources they need from others and voluntarily provide them with the resources they need. Cropanzano ${ }^{31}$ integrates the core ideas of previous social exchange theories and points out that social exchange principles include principles of reciprocity, rules of exchange, and negotiated rules. Principles of reciprocity is the most important exchange principle, that is, people tend to return the good deeds from others (sometimes bad things). ${ }^{32}$ According to the nature of reciprocity in exchange, it can be distinguished into "interdependent exchanges, folk belief, moral norm and individual orientation". ${ }^{31}$ Cropanzano et $\mathrm{al}^{32}$ once again concludes that the behavior mode of social exchange has three common features, namely the initial treatment of the actor to the target individual, the reciprocal response of the target individual to the actor (such as attitudes and behaviors), and the formation of relationship. 
Conservation of resources theory originally proposed by Hobfoll ${ }^{33}$ to explain the psychology and behavioral response of individuals in the face of pressure or not under pressure. This theory holds that people will strive to maintain, protect and construct their own precious resources to avoid the threat of resource loss. ${ }^{33}$ Resources are things perceived by individuals that help to achieve their goals. ${ }^{34}$ With the gradual development and improvement of the theory, Hobfoll $\mathrm{et}^{\mathrm{al}^{25}}$ summarizes and revisions conservation of resources theory, concluding one hypothesis, five principles and three inferences. One hypothesis is that individuals (groups) have the tendency to strive to acquire, reserve, cultivate and protect resources they cherished; five principles include "primacy of loss, resource investment, gain paradox, resource desperation, resource caravans and resource caravan passageways"; three inferences include "initial resource effect, resource loss cycles, resource gain spirals". ${ }^{18}$

\section{SSG and IB}

SSG refers to the quality of personal relationship established between leaders and employees through non-workrelated activities in non-working hours to achieve personal goals. ${ }^{10}$ IB is employees spontaneously create, introduce and apply new ideas in their work role, team or organization to benefit the role performance, team or organization. ${ }^{35}$

Based on social exchange theory, the SSG should be beneficial to employee IB. First, employees with close SSG can get support from leaders, such as career development opportunities, high-quality work tasks and resources, ${ }^{11}$ encourage and support employees' personal development. At this time, employees will think that they are more important than others, will be more willing to engage in challenging work, which is more conducive to stimulating employees' creativity. ${ }^{36}$ Second, based on the principle of reciprocity, employees will also show behaviors that are beneficial to the development of the team and organization to repay the trust and support of leaders. In existing empirical studies, the trust and support of leaders have been proved to be beneficial to employees' perceptions, attitudes and behaviors. For example, Charoensukmongkol and Puyod ${ }^{37}$ illustrate that the transformational leadership that encourages, supports, and empowers employees is often beneficial to employees' work-life balance, knowledge sharing, IB, and reduces the role ambiguity of employees. Guang and Charoensukmongkol $^{38}$ suggest that expatriate leaders with higher cultural intelligence are often regarded as caring and supportive leaders by local employees, which makes employees show commitment and IB to repay. Therefore, the following hypothesis is put forward. Hypothesis 1: SSG is positively associated with IB.

\section{The Mediating Role of JS}

JS is the overall evaluation and attitude of individuals towards their own work. ${ }^{39}$ Hackman $^{40}$ believes that JS especially refers to "satisfaction with opportunities for personal growth and development on the job".

This paper holds that SSG not only directly affects employee IB but also indirectly affects employee IB through JB. Based on social exchange theory, the more time employees engage in social networks, the higher the return they get from engagement (more satisfactory job opportunities). ${ }^{12}$ Leaders will also trust employees with good relationships and create more development opportunities and promotion channels for them, ${ }^{41}$ thereby enhancing employees' JS. Employee with high JS will enhance the job embeddedness, which in turn stimulates employees' vitality to generate, disseminate and implement innovative ideas. ${ }^{24}$ In addition, when employees feel satisfied with their work, based on the principle of reciprocity, employees will also make behaviors (innovative) that are beneficial to the development of the team and organization. Existing empirical studies also support this logic. For example, Li et $\mathrm{al}^{42}$ argue that SSG can positively affect employee JS. Anser et $\mathrm{al}^{43}$ shows that JS can motivate employees' positive behavior and improve organizational performance. Tang et $\mathrm{al}^{44}$ suggest that JS can positively affect employee IB. Although few empirical studies examined JS mediated the effect of SSG on employee IB, Chung and $\mathrm{Kim}^{45}$ note that workplace ostracism can reduce employee JS and then affect their IB, which can be used as a complementary basis for this paper. Therefore, the following hypothesis is put forward. Hypothesis 2: JS will mediate the effect of SSG and IB.

\section{The Mediating Role of OC}

OC refers to a mindset or psychological state, that is, the feelings and/or beliefs of the relationship between employees and the organization, including affective commitment, continuance commitment, and normative commitment. ${ }^{46}$

This paper holds that SSG can also indirectly affect employee IB through OC. Based on social exchange theory, employees with close SSG have a stronger sense of belonging and responsibility as members of the 
organization. ${ }^{11}$ They will receive incentives from leaders, such as job vision and job security. ${ }^{11}$ At this time, employees will show more commitment to repay the preferential treatment given by leaders. ${ }^{47}$ In addition, leaders also tend to give higher performance ratings to such employees, thereby enhancing their loyalty to leaders. ${ }^{48}$ Employees' OC will promote employee IB. This is because based on the principle of reciprocity, employees with a strong sense of belonging and responsibility recognize the leadership and team more ${ }^{49}$ and believe that they are responsible and strive to build the team, and then they will actively exert their subjective initiative $\mathrm{e}^{50}$ and solve existing problems creatively. In order to obtain material and spiritual returns, employees often increase their investment, showing a high degree of innovation. ${ }^{51}$ Existing empirical studies have found that SSG has positively effects on employee OC. ${ }^{12,52}$ Employees' commitment to the organization promotes employee IB, ${ }^{49}$ improves employee performance, ${ }^{53}$ and reduces employee turnover and cyberloafing behavior. ${ }^{54}$ Although few empirical studies examined OC mediated the effect of SSG on employee IB, this paper finds that $\mathrm{OC}$ plays a mediating role in the relationship between leadership style and employee IB. For example, $\mathrm{Gu}$ et $\mathrm{al}^{55}$ note that leadership (a supporting element of trust between leaders and employees) inspires employees to actively generate new ideas by influencing employees' OC. Iqbal et $\mathrm{al}^{56}$ show that entrepreneurial leadership encourages employees to participate in decision-making, enhances their emotional commitment, and in turn motivates employees to participate in innovative activities. Therefore, the following hypothesis is put forward. Hypothesis 3: OC will mediate the effect of SSG and IB.

\section{The Joint Mediation Effect of JS and OC}

Strong OC will make employees believe and accept the organization's goals and values, and are willing to help the organization achieve its goals in novel ways ${ }^{4}$ and stimulate employees' innovation. Based on the principle of reciprocity, it will also encourage employees to adopt innovative ways to obtain more performance to repay leaders or organizations. Porter et $\mathrm{al}^{57}$ believes that JS, as an attitude towards work, is closely related to factors such as salary and promotion opportunities, and JS responds to these factors faster than OC. Based on social exchange theory, employees who are satisfied with their work may be attached to the organization and ultimately increase their level of commitment. ${ }^{58}$ Existing empirical studies have also proved that JS can promote employee OC. ${ }^{12,44}$ Since employees' JS often benefits from maintaining a good and mutually beneficial relationship with their leaders. ${ }^{12}$ For example, leaders who have a close relationship with employees will break through the routine and give employees more resources, ${ }^{41}$ thereby enhancing employee JS. The study of Cheung et $\mathrm{al}^{12}$ also proves that SSG can affect employee OC through employee JS. However, participants in Cheung et $\mathrm{al}^{12}$ study originated from manufacturing enterprises, which takes enterprise profitability as the main goal. Therefore, whether this research conclusion can be applied to employees of enterprises in different fields (such as scientific and technological enterprises with technology $R \& D$ as the main goal) needs to be further verified. In addition, based on the previous studies and inferences of this paper, we believe that SSG should have an impact on employees' IB through JS and OC, and put forward the following hypothesis. Hypothesis 4: JS and OC will joint mediate the effect of SSG and IB.

\section{The Moderating Effect of PS}

PS is an individual's perception of the consequences of interpersonal risks in a work situation. ${ }^{59}$ It is a belief that people will not be harmed, rejected or punished when they come up with new ideas, seek feedback and report mistakes. ${ }^{26}$

Based on conservation of resources theory, close SSG (such as the trust, care and resources that employees receive from leaders) is an important social resource for employees, ${ }^{17}$ and employees will work hard to protect and retain this resource. PS can also be regarded as a kind of work resources, that is, interpersonal and social relationship resources. ${ }^{29}$ Based on the principle of resource caravans and resource caravan passageways, the resources owned by both individuals and organizations are not independent of each other, but will affect each other like the moving "caravan". 60 Therefore, SSG and PS as resources should affect each other. Based on inferences of initial resource effect and resource gain spirals, employees with high PS resources can obtain the support, trust and encouragement of team members, and do not worry about being rejected or punished when sharing and making suggestions. For example, if employees have close SSG resources at the same time, the possibility of resource loss will be further reduced and they will be more able to obtain new resources. ${ }^{25}$ At this time, employees should be more satisfied with their work. On the contrary, employees with low PS resources will be suspicious, jealous, and mistrust of each other, and even lead to vicious competition. ${ }^{61}$ Even if they have close SSG resources, they obtain relatively few initial resources, are more vulnerable to resource loss, and their ability to obtain 
new resources is relatively weak, ${ }^{25}$ which should not be conducive to enhancing employee JS. Therefore, the following hypothesis is put forward. Hypothesis 5: PS will moderate the positive relationship between SSG and JS, that is, the higher the PS, the stronger the relationship between them, and the lower the PS, the weaker the relationship between them.

At the same time, based on the above inferences, employees who have both high PS and SSG resources should enhance their JS, which will strengthen their sense of belonging and responsibility to the organization. In return, employees will strive to show behaviors (innovation) beneficial to the development of the team and organization. However, for employees with low PS resources, the influence of SSG on employee JS will be weakened, which will reduce employee's sense of belonging and responsibility to the organization, and it is not conducive to employees to produce more IB. Therefore, the following hypothesis is put forward. Hypothesis 6: PS will moderate the joint mediation effect of JS and OC in the relationship between $\mathrm{SSG}$ and employee IB, that is, the higher the PS, the stronger the joint mediation effect, and the lower the PS, the weaker the joint mediation effect.

To sum up, the conceptual model of this paper was constructed (Figure 1).

\section{Materials and Methods}

\section{Participants}

The participants were employees from scientific and technological enterprises in China. The reason for choosing scientific and technological enterprises is that scientific and technological enterprises are technology-oriented. Only by increasing R\&D investment, increasing the number of patents and improving the technical innovation capabilities of employees can they continue to grow in an increasingly fierce external competition environment. ${ }^{62}$ Previous studies have also found that employee IB is related to scientific and technological enterprises. $^{63}$

Cross-sectional data collection came from the selfadministered questionnaire survey created on the software "Wenjuanxing". This paper has been approved by the ethics committee of the first author's organization. First of all, employees from scientific and technological enterprises were recruited by researcher and assistant through online publishing information, and snowball sampling were carried out. The recruited employees came from Beijing, Shandong, Jiangsu and other places of China, including electronics, R\&D, integration and maintenance, communication and other departments. Then, the purpose, requirements and importance of research were introduced to participants. Participants were also told that the survey was anonymous, and the results of the survey were kept confidential so as to eliminate responsebias such as social desirability, falsification, and regular responses. Next, the link of "Wenjuanxing" was sent to participants. The content of the link includes demographic information and questionnaires. Among them, the response-bias identification item was set in the questionnaire (such as "To ensure the quality of the answer, please select 'relatively agree'

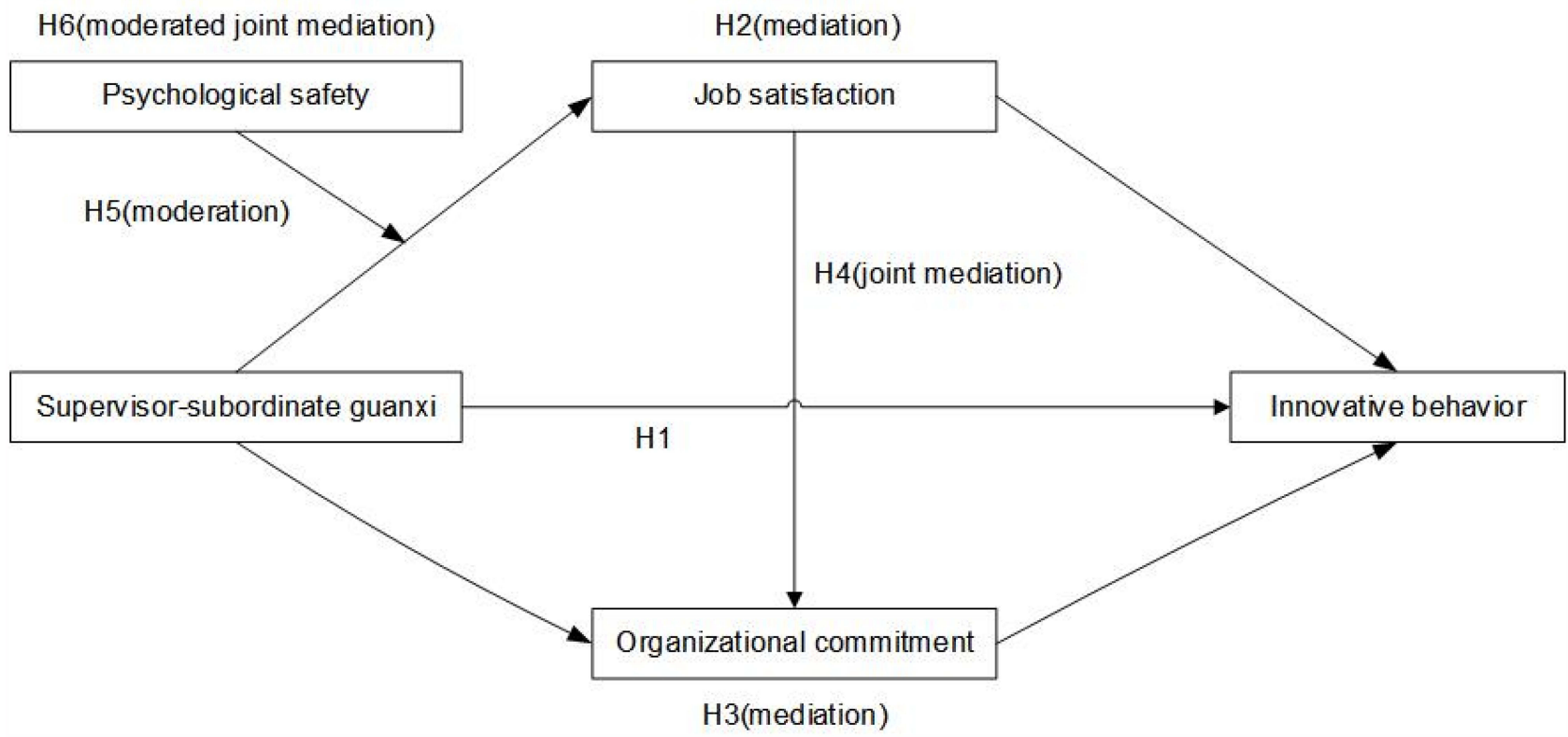

Figure I The conceptual model. 
for this question") to resist the participants' response-bias again. Fill in the questionnaire and submit it online was equivalent to obtaining the informed consent. The process of data collection took approximately 2 months. Finally, a total of 38 enterprises and 246 questionnaires were involved. Excluding invalid questionnaires (regular answers, the same answer options, etc.), 30 enterprises and 207 questionnaires were obtained with an effective recovery rate of $84.1 \%$. Among all participants, $54.1 \%$ were male, $45.9 \%$ were female. $34.8 \%$ were 25 years old and below, $53.1 \%$ were 26 to 35 years old, $12.1 \%$ were 36 years old and above. $22.7 \%$ were junior college and below, $52.7 \%$ were undergraduate, $24.6 \%$ were master and above. $69.6 \%$ were ordinary employees, $30.4 \%$ were managers (grassroots, middle and senior).

\section{Measures}

In this paper, except for IB scale, the measurement items use 7-point Likert scoring, ranging from one ("never") to seven ("always"), and all other scales use 5-point Likert scoring, ranging from one ("strongly disagree") to five ("strongly agree").

\section{SSG}

This is a six-item scale that assesses SSG. ${ }^{10}$ An item includes "My supervisor invites me to his/her home for lunch or dinner". Alpha was 0.9.

\section{JS}

This is a three-item scale that measures JS. ${ }^{64}$ A sample item includes "I am generally satisfied with the work in this position". Alpha was 0.9 .

\section{OC}

This is an eighteen-item scale assessing $\mathrm{OC},{ }^{46}$ which includes three dimensions: affective commitment, continuance commitment, and normative commitment. Affective commitment including "I have an emotional attachment to this enterprise". Continuance commitment including "Leaving this enterprise, I have no other jobs to choose from". Normative commitment including "If I leave this enterprise now, I will feel guilty". Alpha was 0.9.

\section{PS}

This is a seven-item scale measuring PS. ${ }^{26}$ Sample items such as "If you make a mistake in the team, it is often held against you (reverse scored)" and "It's safe to take risks in this team". Alpha was 0.6 , and the reliability is general. However, for the 5-9-item scale, 0.6 is also acceptable. ${ }^{65}$

\section{IB}

This is a nine-item scale that assesses $I B,{ }^{35}$ which includes three dimensions: idea generation, idea promotion, and idea realization. Idea generation including "Generate new ideas for difficult problems". Idea promotion including "Acquiring approval for innovative ideas from other members". Idea realization including "Systematically introduce innovative ideas into the work environment". Alpha was 0.9.

\section{Control Variables}

We utilized gender, age, education level, and hierarchical level as a control variable as gender, age, education level ${ }^{5,66}$ and hierarchical level ${ }^{67}$ have been shown to relate to IB.

\section{Analysis}

SPSS 24.0 and Mplus 8.3 were used for data analysis. First, mplus was used to conduct confirmatory factor analysis on the questionnaire. Harman's one-factor test method was used to test common method bias. Second, descriptive statistics and variable intercorrelations were carried out between variables. Finally, SPSS PROCESS macro was used for hypothesis testing.

\section{Results}

\section{Confirmatory Factor Analysis}

Mplus 8.3 was used to conduct confirmatory factor analysis, and the results are shown in Table 1. Since the fitting data of the initial five-factor model was not very well $\left(\chi^{2}\right)$ $d f=2.267, \quad \mathrm{p}<0.001, \quad \mathrm{RMSEA}=0.078, \quad \mathrm{CFI}=0.872$, $\mathrm{TLI}=0.857, \quad \mathrm{SRMR}=0.079$ ), the model was modified according to the modification indices reported by the software. ${ }^{68}$ The modified data fitting effect was good $\left(\chi^{2}\right)$ $d f=1.981, \quad \mathrm{p}<0.001, \quad \mathrm{RMSEA}=0.069, \quad \mathrm{CFI}=0.901$, $\mathrm{TLI}=0.890, \mathrm{SRMR}=0.074$ ), and the five-factor model is significantly better than other models, indicating that the measurement scale has good discriminant validity.

\section{Common Method Bias Test}

Harman's one-factor test method was used to perform principal component factor analysis on all items of the research variables, and the first principal component before rotation was $32.268 \%$, which was lower than the critical standard of $40 \%$, indicating that there was no common method deviation. 
Table I Confirmatory Factor Analyses

\begin{tabular}{|l|l|l|l|l|l|l|}
\hline Factors & $\chi^{2}$ & df & RMSEA & CFI & TLI & SRMR \\
\hline One-factor model: SSG+JS+OC+IB+PS & 1967.278 & 350 & 0.149 & 0.518 & 0.480 & 0.134 \\
Two-factor model: SSG+PS, JS+OC+IB & 1578.897 & 349 & 0.130 & 0.634 & 0.603 & 0.136 \\
Three-factor model: SSG+PS, IB, JS+OC & 1058.438 & 347 & 0.100 & 0.788 & 0.769 & 0.110 \\
Four-factor model: SSG, JS+OC, IB, PS & 881.120 & 344 & 0.087 & 0.840 & 0.824 & 0.082 \\
Five-factor model: SSG, JS, OC, IB, PS & 770.638 & 340 & 0.078 & 0.872 & 0.857 & 0.079 \\
\hline
\end{tabular}

Abbreviations: RMSEA, root-mean-square error of approximation; CFI, comparative fit index; TLI, Tucker-Lewis index; SRMR, standardized root mean square residual; SSG, supervisor-subordinate guanxi; JS, job satisfaction; OC, organizational commitment; PS, psychological safety; IB, innovative behavior.

\section{Non-Response Bias}

Lower response rate can easily cause scholars to worry about non-response bias. In order to reduce non-response bias, we have taken measures before and after the questionnaire collection process. Before the questionnaire collection process, we set up clear and organized questionnaire items on the "Wenjuanxing" platform to help participants answer questions easily. Participants who are interested in the research were recruited online, and the research purpose and requirements were introduced in detail so as to improve the response rate of participants as much as possible. The response rate in this paper was $84.1 \%$ ( $>50 \%$ ), which can be used as a reference for lower non-response bias. After the questionnaire collection process, participants were divided into early participants and late participants as responders and non-responders, respectively. ${ }^{69}$ The problem of nonresponse bias was solved by comparing the gender variable of early participants and late participants, as well as early participants and all participants. ${ }^{70,71}$ In this paper, 125 were early participants and 82 were late participants. The chi-square test of early participants and late participants showed that they had no significant difference in gender variable $(p>0.05)$. This result was also verified in the chi-square test of early participants and all participants. Therefore, the possibility of non-response bias can be ruled out.

\section{Descriptive Statistics and Variable Intercorrelations}

Table 2 shows the descriptive statistics and variable intercorrelations. It can be seen that SSG, JS, OC, PS and IB are all positively correlated.

\section{Hypothesis Testing}

SPSS PROCESS macro compiled by Hayes ${ }^{72}$ was used for hypothesis testing, and the control variables (gender, age, education level and hierarchical level) entering the equation were virtualized $(0,1)$. Bootstrapping method was used for repeated sampling 5000 times to obtain the standard error and $95 \%$ confidence interval of parameter estimation.

First, model 4 in PROCESS was selected to test the single mediating role of JS and OC between SSG and employee IB. As shown in Table 3, SSG has a significant positive impact on IB $\left(\beta_{\mathrm{JS}}=0.172, \mathrm{p}<0.05\right.$; $\left.\beta_{\mathrm{OC}}=0.173, \mathrm{p}<0.05\right)$, supporting hypothesis 1 . The effect of SSG on JS was significant $(\beta=0.270, p<0.001)$, and JS was significantly positively related with IB $(\beta=0.449$, $\mathrm{p}<0.001)$. After adding JS, the mediation effect value was 0.121 , and the $95 \%$ CI was [0.059, 0.196], excluding 0 , indicating that JS plays a partial mediating role in the influence of SSG on IB. Hypothesis 2 was verified. SSG has a significant positive effect on $\mathrm{OC}(\beta=0.273, \mathrm{p}<0.001)$, and $\mathrm{OC}$ has a significant positive effect on IB $(\beta=0.442$,

Table 2 Means, Standard Deviations and Correlations of Variables

\begin{tabular}{|l|l|l|l|l|l|c|}
\hline Variables & M & SD & I & $\mathbf{2}$ & $\mathbf{3}$ & $\mathbf{4}$ \\
\hline SSG & 3.16 & 0.87 & - & & & \\
JS & 3.89 & 0.68 & $0.34 I^{* * *}$ & - & & \\
OC & 3.53 & 0.66 & $0.386^{* * *}$ & $0.620^{* * *}$ & - & \\
PS & 3.55 & 0.52 & $0.257^{* * *}$ & $0.47 I^{* * *}$ & $0.392^{* * *}$ & - \\
IB & 4.86 & 0.87 & $0.332^{* * *}$ & $0.422^{* * *}$ & $0.405^{* * *}$ & $0.268^{* * *}$ \\
\hline
\end{tabular}

Note: $\mathrm{N}=207, * * * p<0.001$.

Abbreviations: M, mean; SD, standard deviation; SSG, supervisor-subordinate guanxi; JS, job satisfaction; OC, organizational commitment; PS, psychological safety; IB, innovative behavior. 
Table 3 Testing of the Single Mediating Role of JS and OC

\begin{tabular}{|c|c|c|c|}
\hline Variables & Estimate & SE & $95 \% \mathrm{Cl}$ \\
\hline \multicolumn{4}{|l|}{ JS } \\
\hline$S S G \rightarrow I B$ & 0.172 & 0.069 & {$[0.036,0.309]$} \\
\hline$S S G \rightarrow J S$ & 0.270 & 0.056 & {$[0.160,0.381]$} \\
\hline $\mathrm{JS} \rightarrow \mathrm{IB}$ & 0.449 & 0.083 & {$[0.285,0.613]$} \\
\hline$S S G \rightarrow J S \rightarrow \mid B$ & 0.121 & 0.035 & {$[0.059,0.196]$} \\
\hline \multicolumn{4}{|l|}{ OC } \\
\hline$S S G \rightarrow I B$ & 0.173 & 0.071 & {$[0.034,0.312]$} \\
\hline $\mathrm{SSG} \rightarrow \mathrm{OC}$ & 0.273 & 0.053 & {$[0.169,0.377]$} \\
\hline $\mathrm{OC} \rightarrow \mathrm{IB}$ & 0.442 & 0.089 & {$[0.266,0.617]$} \\
\hline $\mathrm{SSG} \rightarrow \mathrm{OC} \rightarrow \mathrm{IB}$ & 0.121 & 0.036 & {$[0.059,0.197]$} \\
\hline
\end{tabular}

Note: $\mathrm{N}=207$.

Abbreviations: $\mathrm{SE}$, standard error $95 \% \mathrm{Cl}$, the lower and upper limits of a confidence interval, respectively; SSG, supervisor-subordinate guanxi; JS, job satisfaction; OC, organizational commitment; IB, innovative behavior.

$\mathrm{p}<0.001)$. After adding $\mathrm{OC}$, the mediation effect value was 0.121 , and the $95 \%$ CI was $[0.059,0.197]$, excluding 0 , indicating that $\mathrm{OC}$ plays a partial mediating role in the influence of SSG on IB. Hypothesis 3 was verified.

Second, model 6 in PROCESS was selected to test the joint mediating role of JS and OC between SSG and employee IB. As shown in Table 4, the total mediation effect value (the sum of the mediation effects of the three mediation paths) was 0.154 , and the $95 \%$ CI was [0.083, 0.239 ], excluding 0 , the effect was significant. The mediating effect of JS in the relationship between SSG and IB was 0.085 , and its $95 \%$ CI was [0.023, 0.159], excluding 0 , the mediating effect was significant. The mediating effect of OC was 0.033, and its 95\% CI was [0.003, 0.080 ], excluding 0 , the mediating effect was also significant. The joint mediating effect of JS and OC in the relationship between SSG and IB was 0.036, and its 95\% $\mathrm{CI}$ is $[0.006,0.076]$, excluding 0 , indicating that SSG can

Table 4 Testing of the Joint Mediating Role of JS and OC

\begin{tabular}{|l|l|l|l|}
\hline Effects & Estimate & SE & $\mathbf{9 5 \% ~ C l}$ \\
\hline Total effect & 0.294 & 0.070 & {$[0.155,0.432]$} \\
Direct effect & 0.139 & 0.070 & {$[0.001,0.277]$} \\
Total indirect effect of & 0.154 & 0.040 & {$[0.083,0.239]$} \\
mediators & & & \\
Indirect effect (JS) & 0.085 & 0.035 & {$[0.023,0.159]$} \\
Indirect effect (OC) & 0.033 & 0.020 & {$[0.003,0.080]$} \\
Indirect effect (JS and OC) & 0.036 & 0.018 & {$[0.006,0.076]$} \\
\hline
\end{tabular}

Note: $\mathrm{N}=207$.

Abbreviations: SE, standard error; $95 \% \mathrm{Cl}$, the lower and upper limits of a confidence interval, respectively; JS, job satisfaction; OC, organizational commitment. influence employee IB through JS and OC, supporting hypothesis 4 .

Next, model 1 in PROCESS was selected to test the moderating effect of PS on the relationship between SSG and JS. The results show that the interactions between SSG and PS has a significant negative impact on JS $(\beta=-0.221, \mathrm{SE}=0.09, t=$ $-2.457, \mathrm{p}<0.05), 95 \% \mathrm{CI}[-0.399,-0.044]$, indicating that PS plays a moderating role in the relationship between SSG and JS. The moderating effect diagram of PS drawn with reference to Aiken et $\mathrm{al}^{73}$ as shown in Figure 2. When the PS is low, SSG has a significant impact on JS $(\beta=0.312, \mathrm{SE}=0.071, t=4.420$, $\mathrm{p}<0.001$ ); when the PS is high, the influence of SSG on JS was not significant $(\beta=0.082, \mathrm{SE}=0.063, t=1.299, \mathrm{p}>0.05)$, indicating that with the decrease of PS, the influence of SSG on JS increases gradually. Hypothesis 5 was not verified.

Finally, model 83 in PROCESS was selected to test the moderating role of PS in the process of series mediation. As shown in Table 5, when the PS is low, the mediating effect of SSG on employee IB through JS and OC was $0.040,95 \%$ CI [0.005, 0.090], excluding 0, the joint mediating effect was significant; when the PS is medium, the indirect effect was $0.025,95 \%$ CI [0.003, 0.057], excluding 0, the joint mediating effect was significant; when the PS is high, the indirect effect was $0.011,95 \%$ CI $[-0.003,0.034]$, including 0 , the joint mediating effect was not significant. The difference between the indirect effect value of joint mediation path when PS is low and when PS is high was -0.030 , $95 \%$ CI $[-0.074,-0.002]$, excluding 0 . In the series mediation model, the coefficient of determination for PS to play a moderating role was $-0.029,95 \%$ CI $[-0.071,-0.002]$, excluding 0 , indicating that the lower the PS, the stronger the joint mediating effect of JS and OC between SSG and employee IB. Hypothesis 6 was not verified.

\section{Discussion}

This paper answered whether and under what factors SSG can influence IB of employees. The results support most of the hypotheses. SSG can positively affect employee IB. JS and OC play not only single mediating role but also chain mediating role in the influence of SSG on IB. PS negatively moderates the influence of $\mathrm{SSG}$ on JS, and also moderates the chain mediating role of JS and OC between SSG and IB.

\section{Theoretical Implications}

First, this paper expands the antecedents of employee IB from the perspective of relationship. LMX originates from the Western cultural background, emphasizing the working 


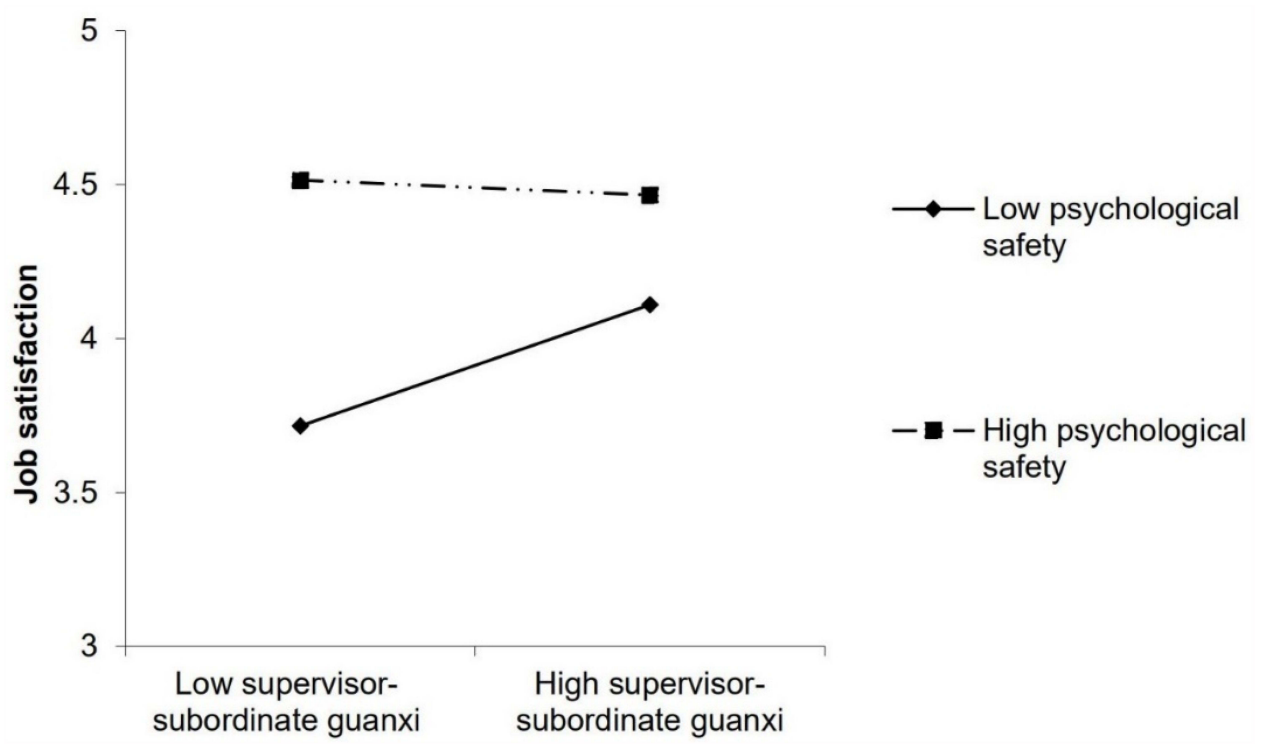

Figure 2 Interaction between supervisor-subordinate guanxi and psychological safety on job satisfaction.

relationship between leaders and employees, and the exchange between leaders and employees is under the norms of fairness and justice, while traditional Chinese culture attaches importance to hierarchical differences and human care. ${ }^{74}$ Employees often make human association with leaders outside the workplace to obtain personal benefits. ${ }^{11}$ Therefore, the concept of SSG is derived, which emphasizes the personal relationship between leaders and employees, and the exchange between leaders and employees is relationship and emotion oriented. ${ }^{11}$ Based on the characteristics of Chinese culture, different from the previous research on LMX, this paper investigates the influence of SSG on employee IB. The results are more localization and effectiveness, and also provide a theoretical basis for Chinese management practices.

Second, this paper breaks the previous mediating idea of studying single variable. In the past, the process mechanism of SSG on outcome variables was mostly limited to a single mediation perspective, ${ }^{12,52}$ lacking the integration of two mediation perspectives, and therefore failed to fully explain the underlying mechanism of the formation of outcome variables. Based on social exchange theory, this paper breaks the mediating idea of single variable in the previous research, constructs a multiple path of "SSG $\rightarrow \mathrm{JS} \rightarrow \mathrm{OC} \rightarrow \mathrm{IB}$ ", and deeply reveals the complex process mechanism of the influence of SSG on employee IB.

Third, this paper broadens the application knowledge of conservation of resources theory. Early conservation of resources theory paid less attention to the interaction between different resources when examining the preservation and maintenance of individual resources. ${ }^{33}$ Hobfoll et $\mathrm{al}^{25}$ summarized and revised the conservation of resources theory and believed that different resources are not independent of each other, but are connected and influenced by each other like a moving fleet. Therefore, we take

Table 5 Testing of Moderated Series Mediation Model

\begin{tabular}{|l|l|l|l|}
\hline Moderators & Estimate & SE & $\mathbf{9 5 \%} \mathbf{~ C l}$ \\
\hline Low PS & 0.040 & 0.022 & {$[0.005,0.090]$} \\
Medium PS & 0.025 & 0.014 & {$[0.003,0.057]$} \\
High PS & 0.011 & 0.009 & {$[-0.003,0.034]$} \\
Medium PS - Low PS (difference) & -0.015 & 0.010 & {$[-0.037,-0.001]$} \\
High PS - Low PS (difference) & -0.030 & 0.019 & {$[-0.074,-0.002]$} \\
High PS- Medium PS (difference) & -0.015 & 0.010 & {$[-0.037,-0.001]$} \\
\hline
\end{tabular}

Note: $\mathrm{N}=207$.

Abbreviations: SE, standard error; $95 \% \mathrm{Cl}$, the lower and upper limits of a confidence interval, respectively; PS, psychological safety. 
close SSG as a valuable resource obtained by employees and PS as an interpersonal relationship resource to investigate the moderating role of PS in the mixed mediating role of SSG and IB. This paper not only broadens the application scope of conservation of resources theory but also responds to the suggestions of Miao et al, ${ }^{11}$ supplements the boundary conditions of SSG on outcome variables, and provides more information for the understanding of SSG on outcome variables.

Fourth, this paper deepens the understanding of the role of PS. Previous studies have mostly found that PS is beneficial to employees' attitudes and behaviors, ${ }^{27,28}$ and when PS was used as a moderating variable, most of them can promote the strength of the relationship between independent variables and dependent variables. ${ }^{75}$ The results of this paper found that the lower the PS, the stronger the indirect effect (JS and OC) of SSG on employees' IB, which is inconsistent with previous research conclusions, but echoes the inference put forward by Edmondson and Lei, ${ }^{59}$ that "PS is not a panacea to solve all challenges in organizational collaboration and learning". PS may also have negative effects or do not have an effect. For example, if PS is too high, employees will be too comfortable, unambitious and content with the status quo, ${ }^{76}$ which is bound to be detrimental to employees' JS and stimulate IB. The results of this paper can help scholars deepen their understanding of the role of PS.

Finally, this paper applied cross-sectional survey is appropriate. Previous studies using cross-sectional survey have mostly condemned the limitations of this survey, such as being far inferior to longitudinal research survey in causal inference and testing mediation effects. ${ }^{44,55}$ However, cross-sectional research is not without its advantages. For example, Spector ${ }^{77}$ note that the cross-sectional survey is more suitable for exploratory research, that is, it is not clear about the expected relationship pattern between variables. The cross-sectional survey is also applicable when it is not clear how long the independent variable affects the dependent variable. ${ }^{77}$ Therefore, this paper explores the theoretical unknown research question of how SSG influence employee IB, and it is more appropriate to apply cross-sectional survey research.

\section{Practical Implications}

First, attach importance to cultivating the intimate relationship between leaders and employees. For example, a harmonious and innovative climate should be created in the enterprise, and a variety of team building activities should be held regularly to deepen the communication and understanding between leaders and employees while deepen the trust and friendship between leaders and employees. Leaders should give humanistic care and respect to employees, encourage employees to propose and implement new ideas, recognize the importance of employees to enterprise development so as to inspire employees to produce more IB to repay the favor of the leader. Employees themselves should also trust and respect leaders, learn from them, and learn to make good use of tasks, learning, career development and other resources provided by leaders to better apply them to innovative performance. Second, attach importance to improving employee JS and OC. For example, leaders should not only provide employees with material guarantees such as salary and benefits, comfortable working environment and learning opportunities, but also give employees spiritual support such as appropriate emotional care, free and open communication, employees can make mistakes, recognition and attention so as to enhance employees' selfconfidence, make employees realize that they are an important member in the development of the enterprise, and then improve their satisfaction with their work, enhance their sense of belonging, responsibility and dedication to the enterprise. Third, pay attention to employees with high sense of PS. For example, leaders can set up performance appraisals to mobilize employees' enthusiasm, prevent employees from in a comfortable state of not enterprising, and avoid the counterproductive effect of SSG.

\section{Limitations and Future Research}

This paper also has some limitations. First, this paper adopts a cross-sectional research method. Although the relationship among various variables can be better investigated, the existence of reverse causality or two-way causality cannot be ruled out. For example, OC can affect employee JS. ${ }^{78}$ Moreover, the cross-sectional research method fails to reflect the dynamic reciprocity process between leaders and employees. ${ }^{11}$ Therefore, longitudinal research or simulation methods can be used to obtain more robust causal inference in the future. Second, this paper only examines the influence of SSG on employees' attitudes and behaviors. Although this social exchange relationship can better reflect the rules of human relations and identity relationships in the Chinese context, previous studies have found that SSG and LMX partially overlap in theoretical and empirical results. ${ }^{11}$ Therefore, future research can incorporate LMX to further test the conceptual model in this paper, and compare it with the results of SSG to deepen the understanding of the two and their 
relationship. Third, this paper only examines the influence of SSG on employee IB under the Chinese cultural background. Whether the research results are applicable to countries with different cultural backgrounds remains to be verified. Smith et $\mathrm{al}^{79}$ found that SSG can be applied to countries with Non-Chinese cultural background (Singapore, Brazil, etc.). Therefore, future research can broaden the regional sources of participants, investigate SSG and IB in different countries, verify the conceptual model of this paper, and deepen the cross-cultural understanding of SSG.

\section{Conclusion}

Based on social exchange theory and conservation of resources theory, this paper proposes and verifies the process mechanism and boundary condition of SSG influencing employee IB. The results show that in the context of Chinese culture, inspiring employees' IB within an enterprise can pay attention to the relationship between leaders and employees, improve employee JS, and strengthen employee OC. At the same time, leaders should also pay attention to employees with high PS and mobilize their enthusiasm and initiative to avoid the counterproductive effect of cultivating SSG.

\section{Abbreviations}

SSG, supervisor-subordinate guanxi; JS, job satisfaction; OC, organizational commitment; IB, innovative behavior; PS, psychological safety; LMX, leader-member exchange.

\section{Data Sharing Statement}

The datasets generated during and/or analyzed during the current study are available from the corresponding author on reasonable request.

\section{Ethical Approval}

The study was reviewed and approved by the ethical committee of School of Economics and Management, China University of Geosciences (Beijing). The study process was in accordance with the guidelines outlined in the Declaration of Helsinki. Informed consent was obtained from all study participants. Confidentiality and anonymity were ensured in this study. All participants were informed that they had the right to withdraw from the study at any time.

\section{Acknowledgments}

The authors thank the study participants and thank Yuechi Sun for his help in data collection.

\section{Author Contributions}

All authors made a significant contribution to the work reported, whether that is in the conception, study design, execution, acquisition of data, analysis and interpretation, or in all these areas; took part in drafting, revising or critically reviewing the article; gave final approval of the version to be published; have agreed on the journal to which the article has been submitted; and agree to be accountable for all aspects of the work.

\section{Disclosure}

The authors report no conflicts of interest in this work.

\section{References}

1. Bos-Nehles AC, Veenendaal AAR. Perceptions of HR practices and innovative work behavior: the moderating effect of an innovative climate. Int $J$ Hum Resour Manag. 2019;30(18):2661-2683. doi:10.1080/09585192.2017.1380680

2. Ratasuk A, Charoensukmongkol P. Does cultural intelligence promote cross-cultural teams' knowledge sharing and innovation in the restaurant business? Asia Pacific J Bus Adm. 2020;12(2):183-203. doi:10.1108/APJBA-05-2019-0109

3. Wang Y, Chen Y, Zhu Y. Promoting innovative behavior in employees: the mechanism of leader psychological capital. Front Psychol. 2021;11:598090. doi:10.3389/fpsyg.2020.598090

4. Yang W, Hao Q, Song H. Linking supervisor support to innovation implementation behavior via commitment: the moderating role of coworker support. J Manag Psychol. 2020;35(3):129-141. doi:10.1108/JMP-04-2018-0171

5. Teng C, Hu C, Chang J. Triggering creative self-efficacy to increase employee innovation behavior in the hospitality workplace. $J$ Creat Behav. 2019;54(1):1-14. doi:10.1002/jocb.419

6. Lv M, Yang S, Lv XY, Zhang L, Chen ZQ, Zhang SX. Organisational innovation climate and innovation behaviour among nurses in China: a mediation model of psychological empowerment. $J$ Nurs Manag. 2021;29:1-9. doi:10.1111/jonm.13381

7. Wang D, Qin Y, Zhou W. The effects of leaders' prosocial orientation on employees' organizational citizenship behavior-the roles of affective commitment and workplace ostracism. Psychol Res Behav Manag. 2021;14:1171-1185. doi:10.2147/PRBM.S324081

8. Schermuly CC, Meyer B, Dämmer L. Leader-member exchange and innovative behavior: the mediating role of psychological empowerment. J Pers Psychol. 2013;12(3):132-142. doi:10.1027/ 1866-5888/a000093

9. Yang Y, Li Z, Liang L, Zhang X. Why and when paradoxical leader behavior impact employee creativity: thriving at work and psychological safety. Curr Psychol. 2021;40(5):1911-1922. doi:10.1007/ s12144-018-0095-1

10. Law KS, Wong CS, Wang D, Wang L. Effect of supervisor-subordinate guanxi on supervisory decisions in China: an empirical investigation. Int J Hum Resour Manag. 2000;11 (4):751-765. doi:10.1080/09585190050075105

11. Miao C, Qian S, Banks GC, Seers A. Human resource management review supervisor-subordinate guanxi: a meta-analytic review and future research agenda. Hum Resour Manag Rev. 2019;30 (2):100702. doi:10.1016/j.hrmr.2019.100702

12. Cheung M, Wu WP, Chan A, Wong M. Supervisor-subordinate guanxi and employee work outcomes: the mediating role of job satisfaction. J Bus Ethics. 2009;88:77-89. doi:10.1007/s10551-0089830-0 
13. Charoensukmongkol P. How Chinese expatriates' cultural intelligence promotes supervisor-subordinate guanxi with Thai employees: the mediating effect of expatriates' benevolence. Int J Cross Cult Manag. 2021;21(1):9-30. doi:10.1177/1470595821996735

14. Han Y, Peng Z, Zhu Y. Supervisor-subordinate guanxi and trust in supervisor: a qualitative inquiry in the People's Republic of China J Bus Ethics. 2011;108(3):313-324. doi:10.1007/s10551-011-1092-6

15. Zhang S, Liang J, Zhang J. The relationship between person-team fit with supervisor-subordinate guanxi and organizational justice in a Chinese state-owned enterprise. Int J Sel Assess. 2019;27 (1):31-42. doi:10.1111/ijsa.12235

16. Liu XY, Wang J. Abusive supervision and organizational citizenship behaviour: is supervisor-subordinate guanxi a mediator? Int J Hum Resour Manag. 2013;24(7):1471-1489. doi:10.1080/09585192.201 2.725082

17. Charoensukmongkol P. Supervisor-subordinate guanxi and emotional exhaustion: the moderating effect of supervisor job autonomy and workload levels in organizations. Asia Pacific Manag Rev. 2021. doi:10.1016/j.apmrv.2021.05.001

18. Kim MS, Koo DW. Linking LMX, engagement, innovative behavior, and job performance in hotel employees. Int J Contemp Hosp Manag. 2017;29(12):3044-3062. doi:10.1108/ijchm-06-2016-0319

19. Gao S. Study on the relationship of leader-member exchange and employees' innovation behavior in high-tech enterprises. Technol Innov Manag. 2015;36(2):147-153. doi:10.14090/j.cnki.jscx.2015.0209

20. Miller L, Miller AF. Innovative work behavior through high-quality leadership. Int J Innov Sci. 2020;12(2):219-236. doi:10.1108/IJIS-042019-0042

21. Wang L, Huang J, Chu X, Wang X. A multilevel study on antecedents of manager voice in Chinese context. Chinese Manag Stud. 2010;4(3):212-230. doi:10.1108/17506141011074110

22. Wu W, Liu J, Shang X. Gain without pay causes lazybones' loss: the influence of formal and informal leader-member relationships on customer service performance. Chinese Manag Stud. 2018;12 (3):634-657. doi:10.1108/CMS-03-2017-0057

23. Blau PM. Exchange and Power in Social Life. New York: John Wiley \& Sons; 1964. doi:10.2307/2091154

24. Niu HJ. Is innovation behavior congenital? Enhancing job satisfaction as a moderator. Pers Rev. 2014;43(2):288-302. doi:10.1108/PR-12-2012-0200

25. Hobfoll SE, Halbesleben J, Neveu JP, Westman M. Conservation of resources in the organizational context: the reality of resources and their consequences. Annu Rev Organ Psychol Organ Behav. 2018;5:103-128. doi:10.1146/annurev-orgpsych-032117-104640

26. Edmondson A. Psychological safety and learning behavior in work teams. Adm Sci Q. 1999;44(2):350-383. doi:10.2307/2666999

27. Moin MF, Omar MK, Wei F, Rasheed MI, Hameed Z. Green HRM and psychological safety: how transformational leadership drives follower's job satisfaction. Curr Issues Tour. 2021;24 (16):2269-2277. doi:10.1080/13683500.2020.1829569

28. Cao F, Zhang H. Workplace friendship, psychological safety and innovative behavior in China: a moderated-mediation model. Chinese Manag Stud. 2020;14(3):661-676. doi:10.1108/CMS-09-2019-0334

29. Bakker AB, Demerouti E. The job demands-resources model: state of the art. J Manag Psychol. 2007;33:309-328. doi:10.1108/02683940 710733115

30. Homans GC. Social behavior as exchange. Am J Sociol. 1958;63 (6):597-606. doi:10.1086/222355

31. Cropanzano R, Mitchell MS. Social exchange theory: an interdisciplinary review. $J$ Manage. 2005;31(6):874-900. doi:10.1177/ 0149206305279602

32. Cropanzano R, Anthony EL, Daniels SR, Hall AV. Social exchange theory: a critical review with theoretical remedies. Acad Manag Ann. 2017;11(1):479-516. doi:10.5465/annals.2015.0099

33. Hobfoll SE. Conservation of resources: a new attempt at conceptualizing stress. Am Psychol. 1989;44(3):513-524. doi:10.1037/0003066X.44.3.513
34. Halbesleben JRB, Neveu JP, Paustian-Underdahl SC, Westman M. Getting to the "COR": understanding the role of resources in conservation of resources theory. J Manage. 2014;40(5):1334-1364. doi:10.1177/0149206314527130

35. Janssen O. Job demands, perceptions of effort-reward fairness and innovative work behaviour. J Occup Organ Psychol. 2000;73 (3):287-302. doi:10.1348/096317900167038

36. Zhou J, Hoever I. Research on workplace creativity: a review and redirection. Annu Rev Organ Psychol Organ Behav. 2014;1 (1):333-359. doi:10.1146/annurev-orgpsych-031413-091226

37. Charoensukmongkol P, Puyod JV. Influence of transformational leadership on role ambiguity and work-life balance of Filipino University employees during COVID-19: does employee involvement matter? Int J Leadersh Educ. 2021;1-20. doi:10.1080/ 13603124.2021.1882701

38. Guang X, Charoensukmongkol P. The effects of cultural intelligence on leadership performance among Chinese expatriates working in Thailand. Asian Bus Manag. 2020;1-23. doi:10.1057/s41291-02000112-4

39. Weiss HM, Nicholas JP, Daus CS. An examination of the joint effects of affective experiences and job beliefs on job satisfaction and variations in affective experiences over time. Organ Behav Hum Decis Process. 1999;78(1):1-24. doi:10.1006/obhd.1999.2824

40. Hackman JR, Oldham GR. Motivation through the design of work: test of a theory. Organ Behav Hum Perform. 1976;16(2):250-279. doi:10.1016/0030-5073(76)90016-7

41. Cheung MFY, Wu W. Participatory management and employee work outcomes: the moderating role of supervisor-subordinate guanxi. Asia Pacific J Hum Resour. 2011;49(3):344-364. doi:10.1177/1038411 111413528

42. Li J, Wang W, Sun G, Jiang Z, Cheng Z. Supervisor-subordinate guanxi and job satisfaction among migrant workers in China. Soc Indic Res. 2018;139:293-307. doi:10.1007/s11205-016-1471-6

43. Anser MK, Ali M, Anwar F, Usman M. Subjective age and job satisfaction: a moderated mediation model of job burnout and chronological age. Front Public Health. 2020;8:62. doi:10.3389/ fpubh.2020.00062

44. Tang Y, Shao YF, Chen YJ, Ma Y. How to keep sustainable development between enterprises and employees? Evaluating the impact of person-organization fit and person-job fit on innovative behavior. Front Psychol. 2021;12:653534. doi:10.3389/fpsyg.2021.653534

45. Chung YW, Kim T. Impact of using social network services on workplace ostracism, job satisfaction, and innovative behaviour. Behav Inf Technol. 2017;36(12):1235-1243. doi:10.1080/0144929X.2017.1369568

46. Meyer JP, Allen NJ. A three-complement conceptualization of organizational commitment. Hum Resour Manag Rev. 1991;1(1):61-89.

47. Tse HHM, Huang X, Lam W. Why does transformational leadership matter for employee turnover? A multi-foci social exchange perspective. Leadersh Q. 2013;24(5):763-776. doi:10.1016/j.leaqua.2013.07.005

48. Wong YT, Ngo HY, Wong CS. Antecedents and outcomes of employees' trust in Chinese joint ventures. Asia Pacific J Manag. 2003;20 (4):481-499. doi:10.1023/A:1026391009543

49. Tang Y, Shao YF, Chen YJ. Assessing the mediation mechanism of job satisfaction and organizational commitment on innovative behavior: the perspective of psychological capital. Front Psychol. 2019;10:2699. doi:10.3389/fpsyg.2019.02699

50. Khaskheli A, Jiang Y, Raza SA, Qureshi MA, Salam J, Salam J. Do CSR activities increase organizational citizenship behavior among employees? Mediating role of affective commitment and job satisfaction. Corp Soc Responsib Environ Manag. 2020;27 (6):2941-2955. doi:10.1002/csr.2013

51. Wang QJ, Wei YF, Li XL. Research on the influence of workplace exclusion on employees' innovative behavior: based on the dual mediation of organizational commitment and organizational identity. Sci Technol Prog Policy. 2020;37(22):134-141. doi:10.6049/ kjjbydc.2019080564 
52. Liu C, Wang N, Liang H. Motivating information security policy compliance: the critical role of supervisor-subordinate guanxi and organizational commitment. Int J Inf Manage. 2020;54(28):102152. doi:10.1016/j.ijinfomgt.2020.102152

53. Khan MAS, Jianguo D, Hameed AA, Mushtaq TUL, Usman M. Affective commitment foci as parallel mediators of the relationship between workplace romance and employee job performance: a cross-cultural comparison of the People's Republic of China and Pakistan. Psychol Res Behav Manag. 2018;11:267-278. doi:10.2147/PRBM.S168542

54. Usman M, Javed U, Shoukat A, Bashir NA. Does meaningful work reduce cyberloafing? Important roles of affective commitment and leader-member exchange. Behav Inf Technol. 2021;40(2):206-220. doi:10.1080/0144929X.2019.1683607

55. Gu H, Duverger P, Yu L. Can innovative behavior be led by management? A study from the lodging business. Tour Manag. 2017;63:144-157. doi:10.1016/j.tourman.2017.06.010

56. Iqbal A, Nazir T, Ahmad MS. Entrepreneurial leadership and employee innovative behavior: an examination through multiple theoretical lenses. Eur J Innov Manag. 2020. doi:10.1108/EJIM-062020-0212

57. Porter LW, Steers RM, Mowday RT, Boulian PV. Organizational commitment, job satisfaction, and turnover among psychiatric technicians. J Appl Psychol. 1974;59(5):603-609. doi:10.1037/h0037335

58. Talukder AK. Supervisor support and organizational commitment: the role of work-family conflict, job satisfaction, and work-life balance. $J$ Employ Couns. 2019;56(3):98-116. doi:10.1002/joec.12125

59. Edmondson AC, Lei Z. Psychological safety: the history, renaissance, and future of an interpersonal construct. Annu Rev Organ Psychol Organ Behav. 2014;1(1):23-43. doi:10.1146/annurev-orgpsych -031413-091305

60. Hobfoll SE. Conservation of resource caravans and engaged settings. J Occup Organ Psychol. 2011;84(1):116-122. doi:10.1111/j.20448325.2010.02016.x

61. Yang X, Li W. Ethical leadership, organizational trust and knowledge sharing behavior: the moderation role of psychological safety. Sci Technol Prog Policy. 2017;34(17):143-147. doi:10.6049/ kjjbydc. 2017050540

62. Chen XH, Ma HL. Impact of tech-innovation on growth: are high-tech SMEs different from non-high-tech SMEs? Stud Sci Sci. 2012;30(11):1749-1760.

63. Dong JJ, Chen GJ. Motivation and mechanism of innovation behavior decision-making in scientific and technological enterprises: verification of traceability and fuzzy set qualitative comparison analysis based on grounded theory. Forum Sci Technol China. 2020; (7):111-119. doi:10.13580/j.cnki.fstc.2020.07.018

64. Hackman JR, Oldham GR. Work Redesign. Massachussetts: AddisonWesley; 1980.

65. Kennedy P, Rooney RM, Kane RT, Hassan S, Nesa M. The enhanced Aussie optimism positive thinking skills program: the relationship between internalizing symptoms and family functioning in children aged 9-11 years old. Front Psychol. 2015;6:504. doi:10.3389/ fpsyg.2015.00504
66. Li C, Makhdoom HUR, Asim S. Impact of entrepreneurial leadership on innovative work behavior: examining mediation and moderation mechanisms. Psychol Res Behav Manag. 2020;13:105-118. doi:10.2147/PRBM.S236876

67. Nedkovski V, Guerci M. When homophilous ties matter: social network brokerage and individuals' innovative behavior. Eur Manag J. 2021;39:755-767. doi:10.1016/j.emj.2021.01.012

68. Feng D, Ji L, Yin Z. Personality, perceived occupational stressor, and health-related quality of life among Chinese judges. Appl Res Qual Life. 2014;9(4):911-921. doi:10.1007/s11482-013-9277-z

69. Armstrong JS, Overton TS. Estimating nonresponse bias in mail surveys. J Mark Res. 1977;14(3):396-402. doi:10.1177/ 002224377701400320

70. Lie HC, Rueegg CS, Fosså SD, Loge JH, Ruud E, Kiserud CE. Limited evidence of non-response bias despite modest response rate in a nationwide survey of long-term cancer survivors: results from the NOR-CAYACS study. $J$ Cancer Surviv. 2019;13(3):353-363. doi:10.1007/s11764-019-00757-x

71. Shiau WL, Yuan Y, Pu X, Ray S, Chen CC. Understanding FinTech continuance: perspectives from self-efficacy and ECT-IS theories. Ind Manag Data Syst. 2020;120(9):1659-1689. doi:10.1108/IMDS-022020-0069

72. Hayes AF. Introduction to Mediation, Moderation, and Conditional Process Analysis: A Regression-Based Approach. New York: Guilford Press; 2013.

73. Aiken LS, West SG. Multiple Regression: Testing and Interpreting Interactions. Newbury Park, CA: Sage Publications; 1991.

74. Ren Z, Yang A, Wang D, Lin Y. Structure models of leader-member relationship (LMR) from the perspectives of cultural differences between China and the West. Acta Psychol Sin. 2014; (9):1355-1377. doi:10.3724/SP.J.1041.2014.01355

75. Erkutlu H, Chafra J. Benevolent leadership and psychological well-being: the moderating effects of psychological safety and psychological contract breach. Leadersh Organ Dev J. 2016;37 (3):369-386. doi:10.1108/LODJ-07-2014-0129

76. Edmondson AC. Psychological safety, trust, and learning in organizations: a group-level lens. Trust Distrust Organ Dilemmas Approach. 2004;(12):239-282. doi:10.2307/2666999

77. Spector PE. Do not cross me: optimizing the use of cross-sectional designs. J Bus Psychol. 2019;34:125-137. doi:10.1007/s10869-01809613-8

78. Vandenberg RJ, Lance CE. Examining the causal order of job satisfaction and organizational commitment. $J$ Manage. 1992;18 (1):153-167. doi:10.1177/014920639201800110

79. Smith PB, Wasti SA, Grigoryan L, et al. Are guanxi-type supervisor-subordinate relationships culture-general? An eight-nation test of measurement invariance. J Cross Cult Psychol. 2014;45(6):921-938. doi:10.1177/0022022114530496
Psychology Research and Behavior Management

\section{Publish your work in this journal}

Psychology Research and Behavior Management is an international, peer-reviewed, open access journal focusing on the science of psychology and its application in behavior management to develop improved outcomes in the clinical, educational, sports and business arenas. Specific topics covered in the journal include: Neuroscience, memory and decision making; Behavior modification and management; Clinical applications; Business and sports performance management; Social and developmental studies; Animal studies. The manuscript management system is completely online and includes a very quick and fair peer-review system, which is all easy to use. Visit http://www. dovepress.com/testimonials.php to read real quotes from published authors. 